\title{
On the Discrete-Continuous Modeling of Rotor Systems for the Analysis of Coupled Lateral Torsional Vibrations*
}

\author{
TOMASZ SZOLC ${ }^{\dagger}$ \\ Institute of Fundamental Technological Research, ul. Świętokrzyska 21, PL-00-049 Warsaw, Poland
}

(Received 23 April 1998; In final form 28 July 1998)

\begin{abstract}
In the paper, dynamic investigations of the rotor shaft systems are performed by means of the discrete-continuous mechanical models. In these models the rotor shaft segments are represented by the rotating cylindrical flexurally and torsionally deformable continuous viscoelastic elements. These elements are mutually connected according to the structure of the real system in the form of a stepped shaft which is suspended on concentrated inertial viscoelastic supports of linear or non-linear characteristics. At appropriate shaft crosssections, by means of massless membranes, there are attached rigid rings representing rotors, disks, gears, flywheels and others. The proposed model enables us to investigate coupled linear or non-linear lateral torsional vibrations of the rotating systems in steady-state and transient operating conditions. As demonstrative examples, for the steam turbo-compressor under coupled lateral torsional vibrations, the transient response due to a blade falling out from the turbine rotor as well as the steady-state response in the form of parametric resonance caused by residual unbalances are presented.
\end{abstract}

Keywords: Rotating machinery dynamics, Discrete-continuous model, Non-linear and parametric lateral torsional vibrations, Numerical simulation

\section{INTRODUCTION}

Dynamic investigations of the rotating machinery have been performed for more than 130 years. Applied for these purposes, appropriate mechanical models of the rotor shaft systems were always essentially dependent on a current development of knowledge in this field as well as on available computational tools. The elementary models consisting of massless shafts with one or two rigid rotors, e.g. the Jeffcott (Föppl) rotor, are characterized by relatively simple mathematical description making possible an application of analytical solutions, but such models are not able to represent all important properties of a real system, as it follows from Tondl (1965), Gryboś (1994) and Nelson (1994). Thus, these models are now usually used only for educational purposes or for investigation of selected particular phenomena of the rotor dynamics described by Tondl (1965), Muszyńska

\footnotetext{
* This paper was originally presented at ISROMAC-7.

${ }^{\dagger}$ Tel.: 826 1281. Ext. 319. Fax: 826 9815. E-mail: tszolc@ippt.gov.pl.
} 
and Goldman (1995) and Bently et al.(1995). For the rotating machinery dynamics an application of more advanced models taking into consideration many rotors and a distribution of the shaft inertia was possible when the first computing devices occurred, as mentioned by Nelson (1994). The manual calculators facilitated performance of dynamic analyses of the rotor shaft systems by means of the transfer matrix method. The advanced transfer matrix techniques enable us to take into consideration many important properties of the rotating machines, i.e. distribution of shaft inertia, gyroscopic effects, support properties, large number of rotors and shaft segments, unbalances and others, but by the use of this method only free and steadystate vibrations can be investigated. A fast development of electronic computers has opened new possibilities of application of multi-disk discrete models of the rotating machines for an analysis of steady-state and transient linear and non-linear vibrations, which has been mentioned by Gryboś (1994), Bently et al. (1995). Nevertheless, the multidisk discrete models with massless shafts have been recently almost completely eliminated by the finite element models, as it follows from Gryboś (1994), Nelson (1994), Ecker et al. (1994). Fine nets of finite elements make possible to model the rotor shaft systems with high accuracy, but they usually lead to entire mechanical models of very many degrees of freedom, i.e. much more than a hundred for onedimensional models and thousands for three-dimensional models. Then, for numerical simulations of non-linear processes it is necessary to reduce the number of degrees of freedom of the considered mechanical models. For this purpose two approaches are commonly applied: the static condensation method and the modal synthesis described by Nelson (1994) and used by Ecker et al. (1994). Both can insert into the investigated process more or less essential inaccuracies, diminishing in this way advantages of the finite element modeling.

The continuous modeling of the rotating machines is usually based on the assumption of a uniform axial distribution of mass and stiffness of the rotor shafts, where inertia of numerous turbine bladed disks as well as viscoelastic properties of the supports and sealings are also regarded as uniformly distributed along the entire shaft length, as described by Tondl (1965), Gryboś (1994), or they have been assumed in the form of appropriate distributions of the Dirac type by Lee and Jei (1988). Such approach enables us to describe motion of the rotor, i.e. lateral or torsional vibrations, by means of one global partial differential equation solved usually with simple boundary conditions. Using this strategy it is not possible to take into consideration all important properties of the real rotating machine. According to the above, the continuous models are seldom applied for the engineering practice and they are usually used only for fundamental studies.

The wave interpretation of vibration processes in the continuous and discrete-continuous models of the rotor shaft systems enables us to avoid disadvantages of the above mentioned ways of modeling for the rotor machines under transient and steady-state torsional vibrations, as it follows from Bogacz et al. (1992). For free lateral vibration analysis the discrete-continuous models in the form of rotating Euler-Bernoulli beam with rigid rotors have been applied by Kim et al. (1989). Forced nonlinear coupled lateral-torsional vibrations were investigated by Szolc (1998) using a discretecontinuous model of the high-speed-train wheelset regarded as a rotating Euler-Bernoulli beam in the form of a stepped shaft with rigid and flexible rotors. In the presented paper a more generalized approach to the discrete-continuous modeling of the rotating systems under coupled lateral torsional vibrations is proposed. This approach is convenient for industrial applications and makes it possible to avoid disadvantages and restrictions typical for the transfer matrix method, finite element method and for the continuous models applied so far.

\section{ASSUMPTIONS}

The subject of considerations in this paper are rotating machines with relatively slender and long 
shafts, i.e. steam turbo-generators, turbo-compressors and rotor machines driven by the electric motors, e.g. pumps, blowers, fans, compressors and others. Shafts of these machines usually have the shape of stepped shafts consisting of several cylindrical or almost cylindrical segments. At their appropriate cross-sections there are attached bladed disks, impellers, gears, coupling disks and others, as shown in Fig. 1(a). In order to build a mechanical model of such system for lateral and torsional vibration analysis, let us assume that in the real stepped shaft one can distinguish $n$ cylindrical segments of lengths $l_{i}$, cross-sectional mass densities $\rho A_{i}$, flexural stiffness $E I_{i}$, torsional stiffness $G J_{0 i}$ and eccentricity distributions of the mass centers of gravity $\delta_{i}(x)$ with the appropriate phase angles $\Gamma_{i}$, where $x$ is the spatial co-ordinate and $t$ denotes time, $i=1,2, \ldots, n$. While using the finite element method,

a)

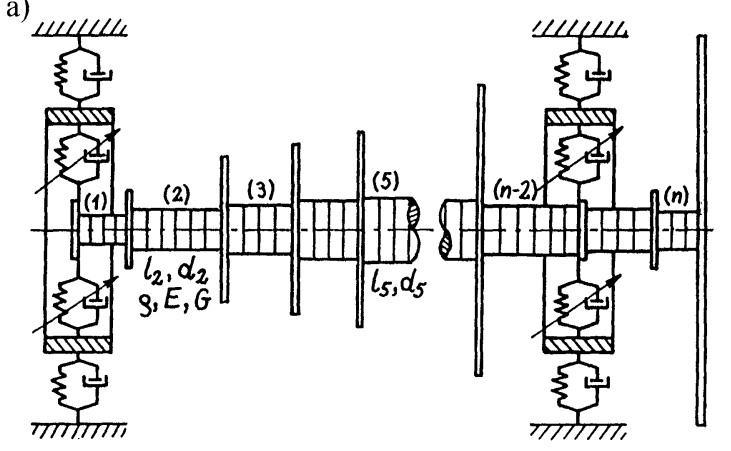

b)

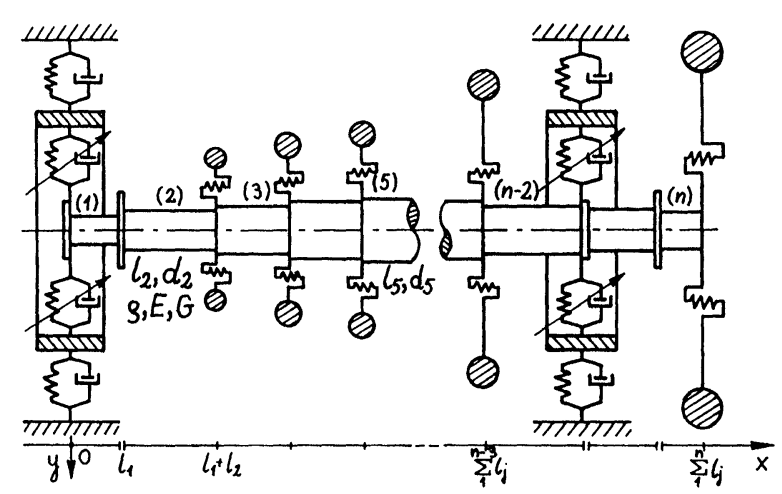

FIGURE 1 (a) Finite element and (b) discrete-continuous models of the rotating machine. in order to take into consideration the continuous distribution of mass along the shaft rotation axis accurately enough, it is necessary to slice each $i$ th cylindrical segment into several beam elements of identical or almost identical lengths, as shown in Fig. 1(a). For example, for the real rotating machine with a stepped shaft of $n=10-30$ cylindrical segments, a division of each into at least 3-5 finite beam elements of 8 degrees of freedom for bending and 2 degrees of freedom for torsion results in an entire discrete mechanical model of $\sim 150-750$ degrees of freedom.

Instead of the traditional finite element method, in this paper the discrete-continuous modeling of the rotor shaft system presented in Fig. 1(a) is proposed. Then, each $i$ th cylindrical segment of the stepped shaft is regarded as flexurally and torsionally deformable continuous viscoelastic element of the same parameters $l_{i}, \rho A_{i}, \quad E I_{i}, \quad G J_{0 i}, \delta_{i}(x), \quad \Gamma_{i}$, $i=1,2, \ldots, n$, as that appointed for a discretization using the finite element method. Similarly as for the one-dimensional finite element models, bladed disks, rotors, impellers, gears, coupling disks, flywheels, etc. can be represented by rigid bodies fixed in appropriate cross-sections of the stepped shaft. In many cases the bending diametral flexibility of the mentioned elements of the rotating machine is high enough to influence the lateral vibrations of the shafts. Then, in the proposed discrete-continuous model these elements are represented by rigid rings attached to the shaft by massless isotropic elastic membranes of the diametral bending stiffness $\mu_{i}$, Fig. 1(b). Each rigid body or rigid ring is characterized by mass $m_{i}$, diametral and polar mass moments of inertia $J_{i}, I_{0 i}$, respectively, radial eccentricity $\varepsilon_{i}$ of the center of gravity with the phase angle $\Delta_{i}$ and by the products of inertia $I_{x y i}, I_{x z i}, I_{y z i}$ expressing its dynamic unbalance, $i=1,2, \ldots, n+1$. In the proposed discrete-continuous model supports of the rotor system are represented in the identical way as for the finite element method, i.e. in the form of discrete oscillators of 2 degrees of freedom each, where in a case of the journal bearings the viscoelastic interaction of the oil film as well as inertialviscoelastic properties of the bearing housings are 
considered, Fig. 1(a) and (b). Beyond external excitations continuously distributed along the cylindrical segments also concentrated external loads as well as concentrated external damping forces and torques can be imposed on appropriate cross-sections of the stepped shaft and on the rigid bodies or rigid rings. The driving and retarding external torques can be described by time functions 'a priori' assumed or by functions of the system response. In the both cases these functions can contain constant average components determining constant rotational speed of the rotor machine.

\section{FORMULATION OF THE PROBLEM}

Further considerations are performed using an orthogonal non-rotating co-ordinate system $O x y z$. The co-ordinate $x$-axis is parallel to the rotation axis of the undeformed rotor shaft with the origin set at the shaft left-most cross-section, as shown in Fig. 1(b). The $y$-axis is vertical, directed downwards, and the $x$-, $z$-axis together determine the horizontal plane.

The shaft is assumed slender enough to omit shear effects in the frequency range corresponding to dynamic interaction between the rotor-shaft system and the bearings. Thus, for "small" lateral vibrations vertical and horizontal motions of circular cross-sections of the $i$ th elastic segment of the stepped shaft are described by means of the rotating Rayleigh beam equation

$$
\begin{aligned}
E I_{i} & {\left[\frac{\partial^{4} v_{i}(x, t)}{\partial x^{4}}+e \frac{\partial^{5} v_{i}(x, t)}{\partial x^{4} \partial t}\right] } \\
& -\rho I_{i}\left[\frac{\partial^{4} v_{i}(x, t)}{\partial x^{2} \partial t^{2}}+2 \mathrm{j} \Omega \frac{\partial^{3} v_{i}(x, t)}{\partial x^{2} \partial t}\right] \\
& +\rho A_{i} \frac{\partial^{2} v_{i}(x, t)}{\partial t^{2}}=\rho A_{i} \delta_{i}(x) \Omega^{2} \exp \left(\Omega t+\Gamma_{i}\right),
\end{aligned}
$$

where $v_{i}(x, t)=u_{i}(x, t)+\mathrm{j} w_{i}(x, t), u_{i}(x, t)$ denotes the lateral displacement in the vertical direction, $w_{i}(x, t)$ denotes the lateral displacement in the horizontal direction, $i=1,2, \ldots, n$, and $\mathrm{j}$ is the imaginary number. The shaft eccentricities $\delta_{i}(x)$ are usually small enough to neglect the coupling effect with torsional vibrations. Torsional motion of the crosssections is described by the following well-known equation:

$$
G\left[\frac{\partial^{2} \theta_{i}(x, t)}{\partial x^{2}}+\tau \frac{\partial^{3} \theta_{i}(x, t)}{\partial x^{2} \partial t}\right]-\rho \frac{\partial^{2} \theta_{i}(x, t)}{\partial t^{2}}=q_{i}(x, t),
$$

where $\theta_{i}(x, t)$ is the angular displacement with respect of the shaft rotational uniform motion with the constant velocity $\Omega$ and $q_{i}(x, t)$ denotes the external torque distribution. The material damping in the shaft is represented by means of the Voigt model, where in Eqs. (1) and (2) $e$ and $\tau$ denote the viscosity coefficients for bending and torsion, respectively.

Equations (1) and (2) are solved with appropriate boundary conditions, which enclose geometrical conditions of conformity for displacements and inclinations of extreme cross-sections of the adjacent elastic segments

$$
\begin{aligned}
v_{i-1}(x, t) & =v_{i}(x, t), \quad \frac{\partial v_{i-1}(x, t)}{\partial x}=\frac{\partial v_{i}(x, t)}{\partial x}, \\
\theta_{i-1}(x, t) & =\theta_{i}(x, t) \\
\text { for } x & =\sum_{j=1}^{i-1} l_{j}, \quad i=2,3, \ldots, n,
\end{aligned}
$$

as well as linear and non-linear equations of equilibrium for the external forces and torques, static and dynamic unbalance forces and moments, inertial, elastic and external damping forces, support reactions and for gyroscopic moments. The equations of boundary conditions corresponding to the shaft cross-sections supported in the anisotropic symmetrical bearings have the following form:

$$
\begin{aligned}
& m_{\mathrm{B} i} \frac{\mathrm{d}^{2} s_{i}}{\mathrm{~d} t^{2}}+d_{2 i y} \operatorname{Re}\left[\frac{\mathrm{d} s_{i}}{\mathrm{~d} t}\right]+\mathrm{j} d_{2 i z} \operatorname{Im}\left[\frac{\mathrm{d} s_{i}}{\mathrm{~d} t}\right] \\
& +k_{2 i y} \operatorname{Re}\left[s_{i}\right]+\mathrm{j} k_{2 i z} \operatorname{Im}\left[s_{i}\right] \\
& +d_{1 i y} \operatorname{Re}\left[\frac{\mathrm{d} s_{i}}{\mathrm{~d} t}-\frac{\partial v_{i}}{\partial t}\right]+\mathrm{j} d_{1 i z} \operatorname{Im}\left[\frac{\mathrm{d} s_{i}}{\mathrm{~d} t}-\frac{\partial v_{i}}{\partial t}\right] \\
& +k_{1 i y} \operatorname{Re}\left[s_{i}-v_{i}\right]+\mathrm{j} k_{1 i z} \operatorname{Im}\left[s_{i}-v_{i}\right]=0,
\end{aligned}
$$




$$
\begin{aligned}
& m_{i} \frac{\partial^{2} v_{i}}{\partial t^{2}}+E I_{i} \frac{\partial^{3} v_{i}}{\partial x^{3}}-\rho I_{i} \frac{\partial^{3} v_{i}}{\partial x \partial t^{2}}-E I_{i-1} \frac{\partial^{3} v_{i-1}}{\partial x^{3}} \\
& +\rho I_{i-1} \frac{\partial^{3} v_{i-1}}{\partial x \partial t^{2}}+2 \mathrm{j} \Omega \rho I_{i} \frac{\partial^{2} v_{i}}{\partial x \partial t}-2 \mathrm{j} \Omega \rho I_{i-1} \frac{\partial^{2} v_{i-1}}{\partial x \partial t} \\
& \quad+d_{1 i y} \operatorname{Re}\left[\frac{\partial v_{i}}{\partial t}-\frac{\mathrm{d} s_{i}}{\mathrm{~d} t}\right]+\mathrm{j} d_{1 i z} \operatorname{Im}\left[\frac{\partial v_{i}}{\partial t}-\frac{\mathrm{d} s_{i}}{\mathrm{~d} t}\right] \\
& \quad+k_{1 i y} \operatorname{Re}\left[v_{i}-s_{i}\right]+\mathrm{j} k_{1 i z} \operatorname{Im}\left[v_{i}-s_{i}\right]=0 \\
& -J_{i} \frac{\partial^{3} v_{i}}{\partial x \partial t^{2}}+E I_{i} \frac{\partial^{2} v_{i}}{\partial x^{2}}-E I_{i-1} \frac{\partial^{2} v_{i-1}}{\partial x^{2}} \\
& \quad+\mathrm{j} I_{0 i} \Omega \frac{\partial^{2} v_{i}}{\partial x \partial t}=0, \\
& -I_{0 i} \frac{\partial^{2} \theta_{i}}{\partial t^{2}}-D_{i} \frac{\partial \theta_{i}}{\partial t}+G J_{0 i} \frac{\partial \theta_{i}}{\partial x}-G J_{0, i-1} \frac{\partial \theta_{i-1}}{\partial x}=0 \\
& \quad \text { for } x=\sum_{j=1}^{i-1} l_{j}, \quad i=k,
\end{aligned}
$$$$
\begin{aligned}
& =I_{0 i} \frac{\partial^{2} \theta_{i}}{\partial t^{2}}+G J_{0, i-1} \frac{\partial \theta_{i-1}}{\partial x}-G J_{0 i} \frac{\partial \theta_{i}}{\partial x} \\
& \quad \text { for } x=\sum_{j=1}^{i-1} l_{j}, \quad i=r,
\end{aligned}
$$

where:

$$
\begin{gathered}
I_{x i}(t)=I_{x y i} \exp \left(\mathrm{j}\left(\pi / 2-\tilde{\Theta}_{i}\right)\right)+I_{x z i} \exp \left(-\mathrm{j} \tilde{\Theta}_{i}\right), \\
\tilde{\Theta}_{i}(x, t)=\Omega t+\theta_{i}(x, t)+\Delta_{i} .
\end{gathered}
$$

From the above equations it follows that the static and dynamic unbalances of the rigid rings or rigid bodies representing rotors, disks, impellers and others couple rotor-shaft lateral vibrations with torsional ones. One can easily remark that the lateral torsional coupling terms in Eqs. (3c) have analogous forms as appropriate coupling terms occurring in equations of motion for a corresponding purely discrete model of the rotor machine described by Tondl (1965) or by Neilson (1992). In Eqs. (3b) and (3c) the symbols $k$ and $r$, $1 \leq k, r \leq n$, denote respectively numbers of the elastic elements following the bearing and disk positions. The quantities $D_{i}$ are the coefficients of absolute damping due to rotational friction in the bearings. Angular displacements of the rigid rings representing masses of the rotors are expressed in (3c) by the complex functions $\varphi_{i}(t)=\phi_{i}(t)+\mathrm{j} \psi_{i}(t)$, where $\phi_{i}(t)$ and $\psi_{i}(t)$ denote the angular displacements in the vertical and horizontal plane, respectively. The concentrated external torques are denoted by $T_{i}(t), i=1,2, \ldots, n+1$.

In order to perform an analysis of free elastic vibrations all the forcing, viscous, non-linear and unbalance terms standing in the boundary conditions (3) have been omitted. Due to truncation of these terms the lateral and torsional vibrations of the rotor shaft system are mutually uncoupled. Thus, the elastic torsional eigenvalue problem can be solved separately.

The equations of motion (1) and (2) are solved using the well-known separation of variables approach

$$
\begin{gathered}
v_{i}(x, t)=V_{i}(x) \cdot T(t), \theta_{i}(x, t)=\Theta_{i}(x) \cdot T(t) \\
\text { for } \sum_{k=1}^{i-1} l_{k} \leq x \leq \sum_{k=1}^{i} l_{k}, \quad i=1,2, \ldots, n,
\end{gathered}
$$


where:

$$
\begin{aligned}
& V_{i}^{I V}(x)+k^{2}\left[k^{2}-2 \Omega \sqrt{\rho / E}\right] V_{i}^{\prime \prime}(x) \\
& -\left(\lambda_{i} k\right)^{4} V_{i}(x)=0, \quad V_{i}(x)=U_{i}(x)+\mathrm{j} W_{i}(x), \\
& \Theta_{i}^{\prime \prime}(x)+(\omega / a)^{2} \Theta_{i}(x)=0, \ddot{T}(t)+\omega^{2} T(t)=0 \\
& \quad \text { and } \lambda_{i}=\sqrt[4]{A_{i} / I_{i}}, k=\sqrt[4]{\omega^{2} \rho / E}, a=\sqrt{G / \rho} .
\end{aligned}
$$

Then, the eigenmode functions are sought in the following form:

$$
\begin{aligned}
V_{i}(x)= & A_{1 i} \sin \left(k \kappa_{i} x\right)+A_{2 i} \cos \left(k \kappa_{i} x\right) \\
& +A_{3 i} \sinh \left(k \chi_{i} x\right)+A_{4 i} \cosh \left(k \chi_{i} x\right), \\
\Theta_{i}(x)= & B_{1 i} \sin \left(\frac{\omega}{a} x\right)+B_{2 i} \cos \left(\frac{\omega}{a} x\right) \\
& \text { for } \sum_{k=1}^{i-1} l_{k} \leq x \leq \sum_{k=1}^{i} l_{k}, \\
\phi_{i}(t)= & \Phi_{i} \exp (\mathrm{j} \omega t), \psi_{i}(t)=\Psi_{i} \exp (\mathrm{j} \omega t), \\
s_{k}(t)= & S_{k} \exp (\mathrm{j} \omega t), T(t)=\exp (\mathrm{j} \omega t),
\end{aligned}
$$

where:

$$
\begin{aligned}
A_{m i} & =A_{m i}^{\mathrm{Re}}+\mathrm{j} A_{m i}^{\mathrm{Im}}, \quad m=1,2,3,4, i=1,2, \ldots, n \\
S_{k} & =Y_{k}+\mathrm{j} Z_{k}, \quad 1 \leq k \leq n,
\end{aligned}
$$

$\kappa_{i}=$

$\sqrt{\frac{1}{2}\left[\sqrt{\left(k^{2}-2 \Omega \sqrt{\frac{\rho}{E}}\right)^{2}+4 \lambda_{i}^{4}}+\left(k^{2}-2 \Omega \sqrt{\frac{\rho}{E}}\right)\right]}$,

$\chi_{i}=$

$\sqrt{\frac{1}{2}\left[\sqrt{\left(k^{2}-2 \Omega \sqrt{\frac{\rho}{E}}\right)^{2}+4 \lambda_{i}^{4}}-\left(k^{2}-2 \Omega \sqrt{\frac{\rho}{E}}\right)\right]}$.

Upon the substitution of solutions (5) into the boundary conditions one obtains separate characteristic equations for the considered eigenvalue problems. These are

$\mathbf{C}(\omega) \cdot \mathbf{D}=\mathbf{0}$ for the lateral free vibrations and

$\mathbf{E}(\omega) \cdot \mathbf{F}=\mathbf{0}$ for the torsional free vibrations, where: $\mathbf{C}(\omega)$ is the characteristic complex matrix $(4 n \times 4 n)$,

$\mathbf{D}=\operatorname{col}\left(A_{11}, A_{21}, A_{31}, A_{41}, A_{12}, A_{22}, \ldots, A_{3 n}, A_{4 n}\right)$,

and $E(\omega)$ is the characteristic real matrix $(2 n \times 2 n)$,

$$
\mathbf{F}=\operatorname{col}\left(B_{11}, B_{21}, B_{12}, B_{22}, \ldots, B_{1 n}, B_{2 n}\right)
$$

Thus, the determination of natural frequencies reduces to the search for values of $\omega$, for which the characteristic determinants of matrices $\mathbf{C}$ and $\mathbf{E}$ are equal to zero. The eigenmode functions are then obtained by solving the characteristic equations (6).

For the forced vibration analysis Eqs. (1) and (2) are solved by means of the Fourier solutions in the form of series in eigenfunctions (5) obtained from (6) for $\Omega=0$ :

$$
\begin{aligned}
u_{i}(x, t) & =\sum_{m=1}^{\infty} U_{i m}(x) \xi_{m}(t), \\
w_{i}(x, t) & =\sum_{m=1}^{\infty} W_{i m}(x) \eta_{m}(t), \\
\phi_{j}(t) & =\sum_{m=1}^{\infty} \Phi_{j m} \xi_{m}(t), \quad \psi_{j}(t)=\sum_{m=1}^{\infty} \Psi_{j m} \eta_{m}(t), \\
\theta_{i}(x, t) & =\sum_{m=1}^{\infty} \Theta_{i m}(x) \vartheta_{m}(t), \\
y_{k}(t) & =\sum_{m=1}^{\infty} Y_{k m} \xi_{m}(t), \quad z_{k}(t)=\sum_{m=1}^{\infty} Z_{k m} \eta_{m}(t), \\
i & =1,2, \ldots, n, j=2,3, \ldots, n+1,1 \leq k \leq n .
\end{aligned}
$$

Using the properties of orthogonality of eigenfunctions (5) obtained for $\Omega=0$ the unknown time functions in series (7) are sought by means of the Lagrange equations of the second order, as it has been done by Szolc (1998). All the temporarily omitted forcing, gyroscopic, viscous, parametric and non-linear terms standing in Eqs. (1) and (2) and in the boundary conditions (3) are regarded here as distributed and concentrated external excitations imposed on the appropriate cross-sections of the rotor shaft or on the appropriate degrees of freedom of the model. The generalized external load $H_{m}(t)$ for 
the given lateral or torsional external distributed excitation $p_{i}(x, t)$ or for the concentrated excitation $P(t)$ is appropriately determined by

$$
H_{m}(t)=\frac{1}{\gamma_{m}^{2}} \sum_{i=1}^{n} \int_{L_{i}}^{L_{i+1}} p_{i}(x, t) X_{i m}(x) \mathrm{d} x
$$

or

$$
H_{m}(t)=\frac{G_{m}}{\gamma_{m}^{2}} P(t), \quad m=1,2, \ldots,
$$

where $X_{i m}(x)$ denotes the respective eigenfunction, $G_{m}=X_{i m}\left(x_{0}\right)$ if the concentrated external excitation $P(t)$ is imposed on the rotor shaft cross-section $x_{0}$, $L_{i} \leq x_{0} \leq L_{i+1}$, or $G_{m}=R_{m}$ if $P(t)$ is imposed on the given generalized co-ordinate $r(t), \quad r(t)=$ $s_{k}(t), \phi_{i}(t), \psi_{i}(t), \quad i=1,2, \ldots, n$, and $L_{i}=\sum_{j=1}^{i-1} l_{j}$. The symbols $\gamma_{m}^{2}, m=1,2, \ldots$, are the coefficients of orthogonality properties of the eigenfunctions in (7). The particular forms of these coefficients can be found in the Appendix. Then, upon appropriate arithmetical rearrangements this approach leads to the system of non-linear and parametric ordinary differential equations for the Lagrange co-ordinates

$$
\begin{aligned}
& \mathbf{M}(\Omega t, t) \ddot{\mathbf{r}}(t)+\mathbf{C}(\Omega, \Omega t, t) \dot{\mathbf{r}}(t)+\mathbf{K}(\Delta v(t)) \mathbf{r}(t) \\
& \quad=\mathbf{F}(t, \Omega t),
\end{aligned}
$$

where:

$$
\begin{aligned}
\mathbf{M}(\Omega t, t) & =\mathbf{M}_{0}+\mathbf{M}_{\mathrm{u}}(\Omega t, t), \\
\mathbf{C}(\Omega, \Omega t, t) & =\mathbf{C}_{0}+\mathbf{C}_{\mathrm{g}}(\Omega)+\mathbf{C}_{\mathbf{u}}(\Omega t, t), \\
\mathbf{K}(\Delta v) & =\mathbf{K}_{0}+\mathbf{K}_{b}(\Delta v(t)) .
\end{aligned}
$$

The symbols $\mathbf{M}_{0}, \mathbf{K}_{0}$ denote, respectively, the constant diagonal modal mass and stiffness matrices, $\mathbf{C}_{0}$ is the constant symmetrical damping matrix and $\mathbf{C}_{\mathrm{g}}(\Omega)$ denotes the skew-symmetrical matrix of gyroscopic effects. The terms of the unbalance effects are contained in the symmetrical matrix $\mathbf{M}_{\mathbf{u}}(\Omega t, t)$ and in the non-symmetrical matrix $\mathbf{C}_{\mathrm{u}}(\Omega t, t)$. Non-linear elastic properties of the journal bearings are described by the symmetrical matrix $\mathbf{K}_{b}(\Delta v(t))$ and the symbol $\mathbf{F}(t, \Omega t)$ denotes the external excitation vector, e.g. due to the unbalance forces. The Lagrange co-ordinate vector $\mathbf{r}(t)$ consists of subvectors of the unknown time functions $\xi_{m}(t)$, $\eta_{m}(t), \vartheta_{m}(t)$ in (7). In order to obtain the system's dynamic response Eqs. (9) are solved by means of a direct integration. The number of Eqs. (9) corresponds to the number of eigenmodes taken into consideration, because the forced lateral and torsional vibrations of the rotor shaft are mutually coupled and thus, according to the appropriate solutions (7), the total number of Eqs. (9) to solve is a sum of all lateral and torsional eigenmodes of the rotor shaft model from the range of frequency of interest.

\section{COMPUTATIONAL EXAMPLES}

The numerical calculations have been performed for the $2000 \mathrm{~kW}$ turbo-compressor with the 5-stage steam turbine, single overhung impeller and with a shaft of the total length $2.0 \mathrm{~m}$ supported on two journal bearings. The bearing span is equal $1.75 \mathrm{~m}$ and the total weight of the entire turbo-compressor rotor shaft system amounts ca. $1680 \mathrm{~kg}$. In this example for the so called 'short' journal bearings anisotropic and symmetrical properties of the oil film have been assumed, which seems to be acceptable for the journal length-to-diameter ratio $L / D \rightarrow 0$. The constant values of the bearing stiffness and damping coefficients are determined using the procedure described by Gryboś (1994) based on the linearized Reynolds' theory. The mechanical model of this turbo-compressor possesses the stepped shaft of $n=9$ continuous cylindrical segments, 4 rigid disks and 6 rigid rings representing rotors, as shown in Fig. 1(b). The bearing positions are indicated in (3b) by $k=1,8$.

\subsection{Free Vibration Analysis}

To obtain a better insight into the dynamic properties of the considered rotating machine, in particular in order to investigate its sensivity to several kinds of vibrations, the free lateral and torsional vibration analysis has been performed. As it was mentioned in Section 3, free elastic lateral and torsional vibrations can be analyzed separately for the proposed model by solving Eqs. (6). 
The free lateral vibration analysis has been made in the frequency range $0-400 \mathrm{~Hz}$ and in the range of shaft rotational velocity $\Omega=0-630 \mathrm{rad} / \mathrm{s}$ in order to investigate the influence of gyroscopic moments on natural frequency values. In Fig. 2 there are depicted the eigenmode functions together with respective natural frequencies obtained for the turbo-compressor rotating at $\Omega=377 \mathrm{rad} / \mathrm{s}$. In these figures the vertical projections of the eigenmodes are plotted by the solid lines and their horizontal projections by the dashed lines. From the results shown in Fig. 2 it follows that in the investigated frequency range the turbo-compressor possesses 10 eigenmodes of lateral vibrations. From the analogous results obtained for $\Omega=0$ not presented here in the form of graphs it follows that when the rotor shaft does not rotate, it vibrates only in the vertical plane in the case of the 2nd, 4th, 6th, 8th and 10th eigenmode. Hovever, for the 1st, 3rd, 5th, 7th and 9th eigenmode the shaft vibrates only in the horizontal plane. For the rotating turbo-compressor the gyroscopic moments couple the rotor shaft motion in the vertical and the horizontal plane, as demonstrated by the shapes of all eigenmode functions in Fig. 2. Moreover, the gyroscopic moments change values of the natural frequencies corresponding to respective eigenmodes. Fig. 3 presents plots of values of the first ten successive lateral eigenfrequencies determined as functions of the rotational speed $\Omega$. From the obtained plots it follows that in the range of $\Omega=0-630 \mathrm{rad} / \mathrm{s}$ the influence of gyroscopic moments on the natural frequency values is negligible only for the third eigenmode. The natural frequency of this eigenmode decreases almost unremarkably within the whole investigated range of $\Omega$. However, the natural frequency values of the remaining eigenmodes are essentially influenced by the gyroscopic effects. The natural frequencies of the 1st, 2nd, 5th, 7th and 9th eigenmode decrease if $\Omega$ increases, while the natural frequencies of the 4th, 6th, 8th and 10th eigenmode increase. These eigenmodes exhibit the backward and forward whirl effects described by Lee and Jei (1988) typical for vibrating rotors suspended on the anisotropic supports.
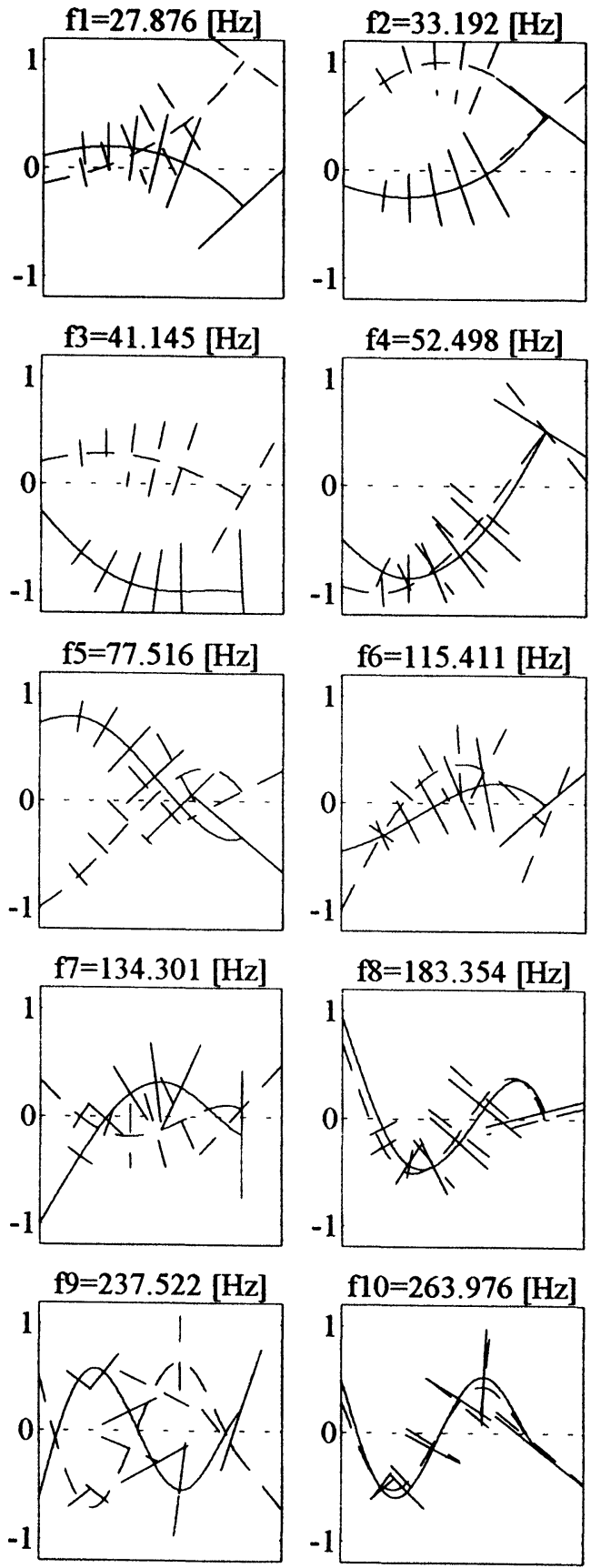

FIGURE 2 Lateral eigenmode functions for the turbocompressor.

The results of the torsional free vibration analysis are presented in Fig. 4. From this figure it follows that in the frequency range $0-400 \mathrm{~Hz}$ three eigenmodes occur for the considered turbo-compressor. 


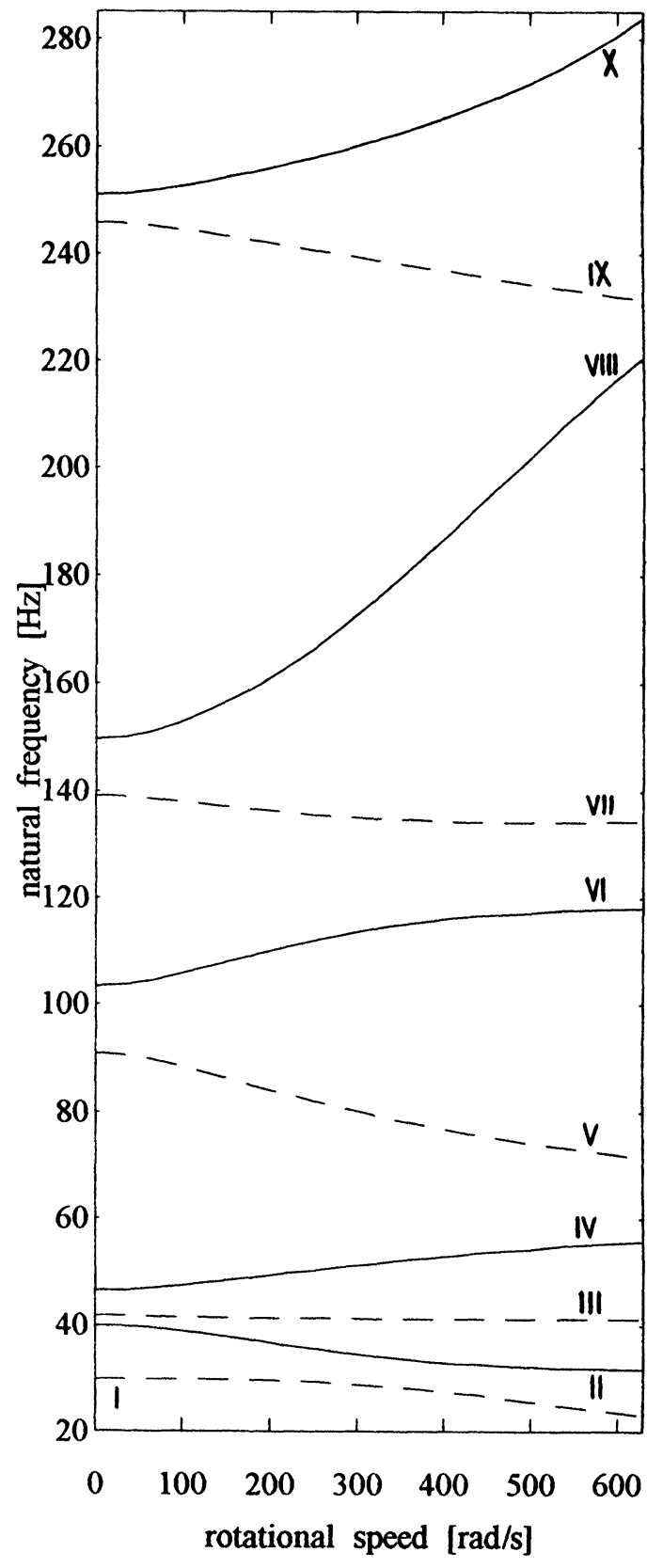

FIGURE 3 Natural frequencies vs. rotational speed.

\subsection{Forced Vibration Analysis}

For forced vibration analysis all constant external loads as well as torsional external excitations imposed on the turbo-compressor have been

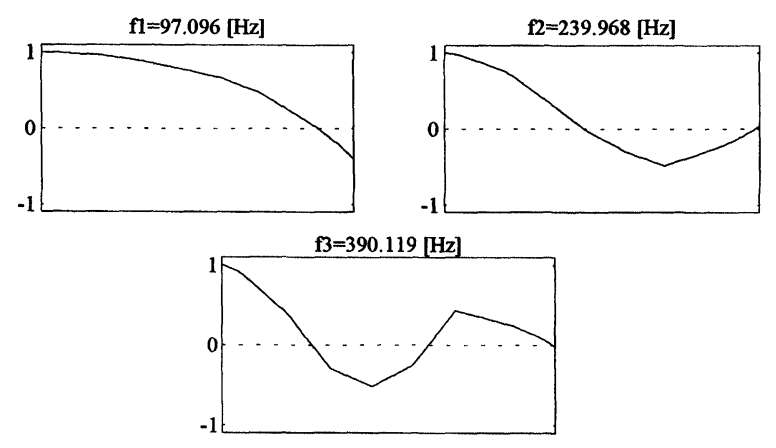

FIGURE 4 Torsional eigenmode functions for the turbocompressor.

neglected. There are assumed static unbalances for which: $\varepsilon_{i}=2.5 \cdot 10^{-4} \mathrm{~m}$ and $\Delta_{i}=0, i=3,4, \ldots, 7$, for the turbine rotors, and $\varepsilon_{10}=2.5 \cdot 10^{-4} \mathrm{~m}$ and $\Delta_{10}=\pi \mathrm{rad}$ for the compressor impeller. Beyond the static unbalances also dynamic ones are assumed, identical for the turbine rotors and compressor impeller, where $I_{x y i}=I_{x z i}=I_{y z i}=$ $0.4 \cdot 10^{-2} \mathrm{kgm}^{2}, i=3,4, \ldots, 7,10$. The rotor shaft unbalance has been omitted assuming $\delta_{i}(x)=0$, $i=1,2, \ldots, 9$, in Eq. (1). For an appropriate finite number of eigenmodes taken into consideration a relatively fast convergence of series (7) assures a sufficiently accurate solution of Eq. (9). For the investigated mechanical system in the frequency range $0-800 \mathrm{~Hz} 18$ lateral and 6 torsional eigenmodes of the rotor shaft system have been considered to solve Eq. (9).

In the first numerical example the system's transient response due to a turbine blade falling out is presented. After $0.75 \mathrm{~s}$ of the operation at constant nominal rotational speed $\Omega=377 \mathrm{rad} /$ $\mathrm{s}=3600 \mathrm{rpm}$ there is assumed the most unfortunate falling out of the heaviest turbine blade from the 5 th disk situated in the neighborhood of the right bearing, Fig. 1. It causes an increase of the mass eccentricity $\varepsilon_{7} 3.5$ times with the unchanged phase angle $\Delta_{7}$. The transient dynamic response is studied in the form of radial vertical and horizontal bearing forces, dynamic torque transmitted by the shaft between the turbine and the compressor impeller as well as in the form of radial displacement orbit of the right bearing journal center, as shown in Fig. 5. The 

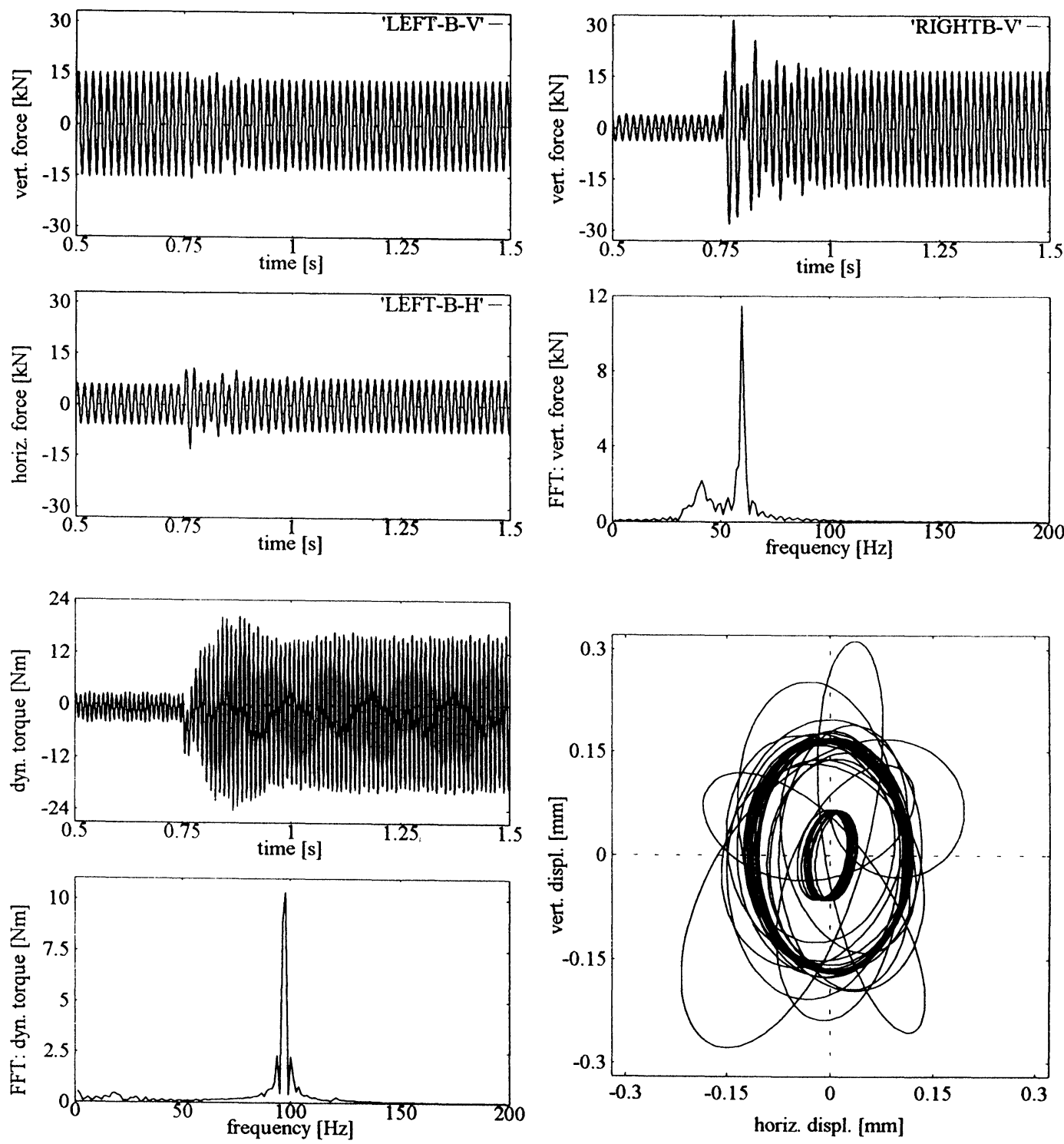

FIGURE 5 Transient dynamic response due to the blade falling out.

rapid increase of system unbalance results in the most severe transient response for the radial vertical force in the right bearing and in much stronger further fluctuation of this force. Then, beyond the fundamental vibrations at frequency $f=\Omega / 2 \pi=$ $60 \mathrm{~Hz}$, also there was excited additional transient component of frequency $\sim 41 \mathrm{~Hz}$ corresponding to the rotor shaft third eigenform, Fig. 2. Consequently, the greater dynamic load of the right bearing results in the "new" larger orbit of its journal center, as shown in Fig. 5. Moreover, the fluctuation of the dynamic torque rapidly increased at the steady frequency $\sim 97 \mathrm{~Hz}$ corresponding to the first torsional eigenform, Fig. 4. The blade falling out causes relatively small changes of fluctuation of the radial vertical and horizontal force in the left bearing, which follows from the respective plots in Fig. 5.

In the second example the influence of static unbalances on the torsional vibration magnitude in 
steady-state operating conditions is investigated. As shown in Fig. 5, for the above mentioned values of $\varepsilon_{i}$ before the blade fall out, the amplitudes of shaft torsional vibrations are very small, i.e. they do not exceed $3 \mathrm{Nm}$. If all eccentricities $\varepsilon_{i}$ are assumed 4 times greater with the same phase angles $\Delta_{i}$, $i=3,4, \ldots, 7,10$, at the nominal rotational speed $\Omega=377 \mathrm{rad} / \mathrm{s}=3600 \mathrm{rpm}$ the amplitudes of vertical force fluctuation in the left bearing are slightly greater than these in the previous case, as it follows from Fig. 6(a). However, the amplitudes of dynamic torque in the shaft between the turbine and the compressor impeller are much greater and they reach $60 \mathrm{Nm}$. When the shaft rotational speed $\Omega$ increases, the system torsional response becomes much more severe to achieve the extreme magnitude at $\Omega=405.3 \mathrm{rad} / \mathrm{s}=3870 \mathrm{rpm}$. Then, the fluctuation amplitude of the dynamic torque is 5 times greater exceeding $300 \mathrm{Nm}$ at frequency $\sim 97 \mathrm{~Hz}$, Fig. 6(b). The amplitudes of the system lateral response have been also increased unproportionally to the rise of excitation amplitudes due to the unbalances. The fluctuation of the vertical force in the left bearing became more than twicely greater at $\Omega=3870 \mathrm{rpm}$, while in comparison with $\Omega=$ $3600 \mathrm{rpm}$ the excitation amplitudes due to the static unbalances $m_{i} \varepsilon_{i} \Omega^{2}$ have increased only 1.16 times. The similar progressive rise of the system lateral response demonstrate the radial displacement orbits of the left and right bearing journals presented in Fig. 6(a) and (b). However, from the analogous orbits for the center of the heavy impeller it follows that for $\Omega=3870 \mathrm{rpm}$ only the vertical displacements are slightly greater and the horizontal ones are almost the same as for $\Omega=$ $3600 \mathrm{rpm}$. The rotational speed $\Omega=3870 \mathrm{rpm}$, for which the extremal dynamic response is observed, corresponds to the unbalance excitation frequency $f=\Omega / 2 \pi=64.5 \mathrm{~Hz} \cong\left(\omega_{b I I}+\omega_{t \mathrm{II}}\right) / 2$, where $\omega_{b \mathrm{II}}=$ $32.823 \mathrm{~Hz}$ denotes the second lateral natural frequency for $\Omega=3870 \mathrm{rpm}$, Fig. 2, and $\omega_{t \mathrm{I}}=$ $97.096 \mathrm{~Hz}$ is the first torsional natural frequency, Fig. 4. Thus, the parametric 'combined' resonance of the first order takes place, which is typical for the rotating systems under coupled lateral torsional vibrations described by equations with periodic coefficients and studied by Tondl (1965) and Neilson (1992).

\section{CLOSING REMARKS}

In this paper transient and steady-state coupled lateral torsional vibrations of the rotating machine were investigated by means of the discrete-continuous mechanical model. Relatively simple geometrical shapes of the real rotor shafts enable us to model in the form of continuous stepped shafts with rigid or rigid-elastically-attached rotors. These models have identical structure as analogous finite element models with the same numerical data, which for the both models results in the same or almost the same level of parameter identification errors. In the case of a classical finite element formulation for the model, taking into consideration non-linear or parametric effects simulations of forced vibrations, usually reduce to direct integration of a set of simultaneous ordinary differential equations, number of which is usually equal to the total number of degrees of freedom, if the wellknown model reduction methods are not used. Then, the direct integration of at least a hundred or more ordinary differential equations results in a huge numerical effort even for powerful modern computers. However, an application of the degree of freedom reduction methods for the finite element models on the one hand significantly minimizes the numerical effort, but on the other hand it can introduce essential errors leading to resultant computational inaccuracies. The discrete-continuous model of the rotating machine proposed in the paper is characterized by the purely analytical mathematical description together with analytical solutions of the Fourier type, which leads strictly to the system of simultaneous non-linear and parametric ordinary differential equations in the Lagrange's co-ordinates, number of which corresponds to the number of all eigenmodes in the frequency range of interest. It is to emphasise that strictly analytical derivation of matrices of these 

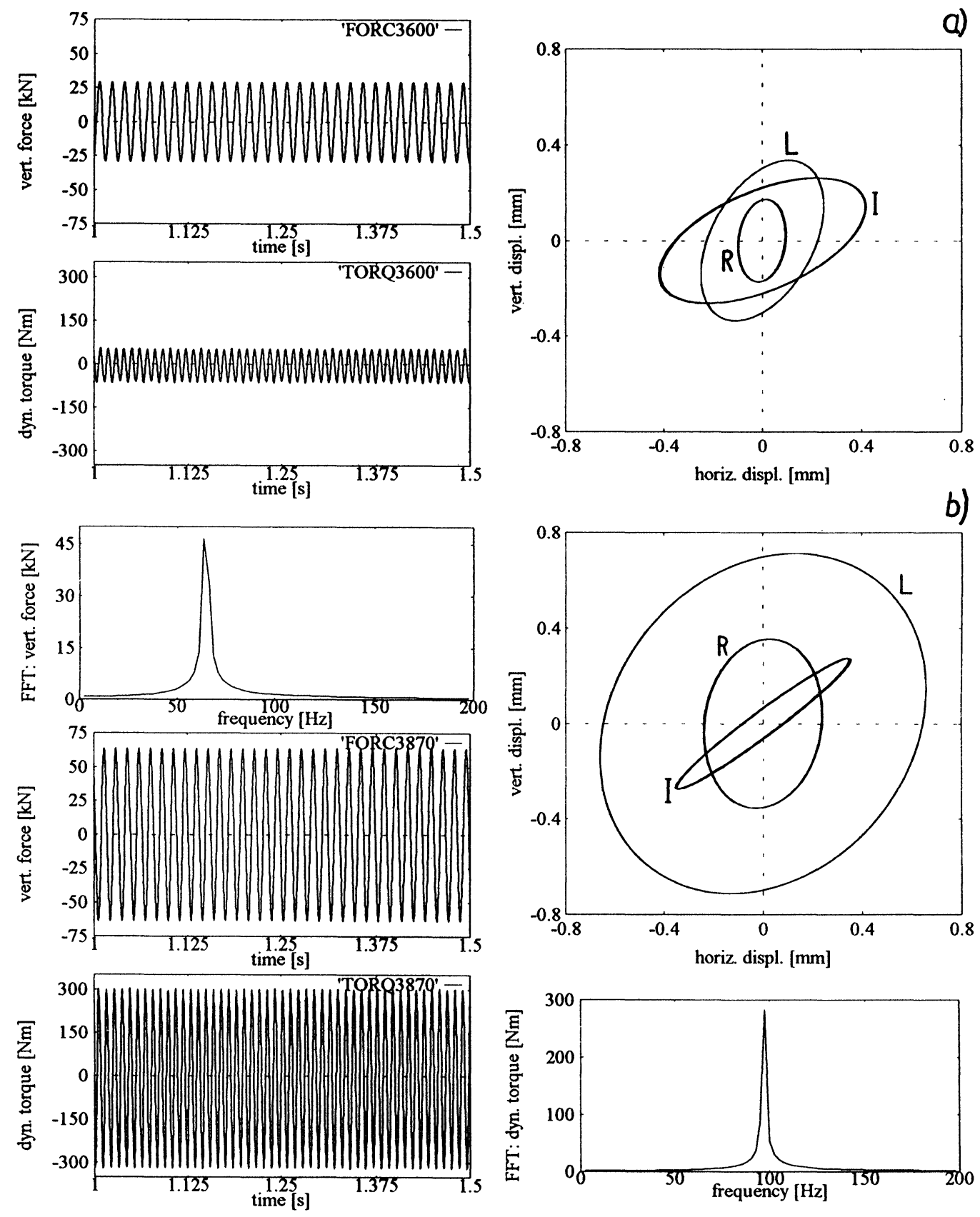

FIGURE 6 Dynamic response in the form of parametric 'combined' resonance: (a) $\Omega=3600 \mathrm{rpm}$; (b) $\Omega=3870 \mathrm{rpm}$.

equations and the mathematically proved fast convergence of the Fourier type solutions in the form of series applied for the discrete-continuous model enable us to expect more accurate results of simulations than those obtained for an analogous finite element model of the identically identified parameters but based on purely numerical approximations of the mathematical description. In the proposed approach a system dynamic response is obtained by means of a direct numerical integration 
of usually anywhere from $10-20$ or at most $20-30$ non-linear or parametric ordinary differential equations, which significantly minimizes the numerical effort. A similar minimization of the numerical effort can be achieved in a case of the finite element method, if the above mentioned reduction algorithms of degrees of freedom are used. However, the ordinary differential equations for the Lagrange's co-ordinates are derived strictly yielding any loss of computational accuracy in the investigated range of frequency in a contradistinction to the ordinary differential equations for the reduced finite element models, which seems to be one of the most advantageous properties of the presented discrete-continuous way of modeling. Moreover, for a given rotor machine the proposed method requires less numerical data to insert for computations, because the discrete-continuous model usually contains a smaller quantity of the cylindrical stepped shaft segments as the total number of beam elements in an analogous finite element model.

The presented numerical examples demonstrate application possibilities of the proposed method for an investigation of practical problems. From the equations of boundary conditions it follows that an increase of the unbalance values, i.e. the eccentricities describing the static unbalances and the products of inertia describing the dynamic ones, causes a proportional rise of the interaction intensity between the lateral and torsional vibrations. This fact has been confirmed by the both numerical examples of the forced vibration analysis. For relatively small static or dynamic unbalances, i.e. for admissible values from the viewpoint of rotor machine exploitation regimes, the coupling between the lateral and torsional vibrations is small enough to analyze them separately. However, a rapid increase of unbalance in the form of a blade falling out or an operation of the badly balanced machine in parametric resonance conditions induce beyond the strong lateral vibrations also severe torsional ones, which can lead to fatigue damage of the rotor shaft material. During an exploitation of each rotating machine one must not exclude such events and thus in many cases an investigation of the coupled by the unbalances lateral torsional vibrations should be regarded as a routine step of the dynamic analysis.

\section{NOMENCLATURE}

$A_{i} \quad$ cross-section area of the $i$ th rotor $\mathbf{C}_{\mathrm{g}}$

$\mathbf{C}_{\mathrm{u}}$

$\mathbf{C}, \mathbf{C}_{0}$

$\mathbf{C}, \mathbf{E}$

$\mathbf{D}, \mathbf{F}$

$d_{m i s}$

$D_{i}$

$e$

$E, G$

$\mathbf{F}(t, \Omega t)$

$H_{m}(t)$

$I_{i}$ shaft segment,

gyroscopic matrix

matrix of the unbalance effects,

damping matrices,

characteristic matrices,

vectors of coefficients standing in the eigenfunctions,

coefficient of damping due to lateral motion in the bearings, coefficient of absolute damping due to rotational friction in the bearings,

bending material viscosity, Young's and Kirchhoff's moduli, vector of external excitations, generalized external load, geometric diametral crosssectional moment of inertia of the $i$ th rotor shaft segment,

$I_{0 i} \quad$ polar mass moment of inertia of the $i$ th rigid ring or rigid body representing rotors or disks,

$I_{x y i}, I_{x z i}, I_{y z i} \quad$ products of inertia of the rigid rings representing rotors,

$J_{i} \quad$ diametral mass moment of inertia of the $i$ th rigid ring or rigid body,

$J_{0 i} \quad$ polar geometric cross-sectional moment of inertia of the $i$ th rotor shaft segment,

$k_{m i s} \quad$ vertical and horizontal stiffness of the bearings,

$\mathbf{K}, \mathbf{K}_{0}, \mathbf{K}_{b} \quad$ stiffness matrices, $l_{i}$ length of the $i$ th rotor shaft segment,

$L_{i} \quad$ length parameter of the rotor shaft, 
$m$

$m_{\mathrm{B} i}$

$\mathbf{M}, \mathbf{M}_{0}$

$\mathbf{M}_{\mathrm{u}}$

$n$

$r(t)$

$\mathbf{r}(t)$

$s_{i}(t)$

$t$

$u_{i}(x, t), w_{i}(x, t)$ bending displacements in the

$V_{i}(x), W_{i}(x) \quad$ eigenfunctions of the bending

$v_{i}(x, t)$

$V_{i}(x)$

$x, y, z$

$y_{k}(t), z_{k}(t)$

$Y_{k}$

$Z_{k}$

$\gamma_{m}^{2}$ vertical and horizontal direction of the $i$ th rotor shaft segment, displacement in the vertical and horizontal direction of the $i$ th rotor shaft segment,

$\delta_{i}(x)$

$\Delta_{i}, \Gamma_{i}$ senting inertia of the bearing housings,

mass matrices,

mass matrix of the unbalance effects,

number cylindrical segments of the rotor shaft,

generalized co-ordinate,

vector of the Lagrange's co-ordinates,

complex generalized co-ordinate describing motion of the bearing housing, complex bending displacement of the $i$ th rotor shaft segment, complex eigenfunction of the bending displacements of the $i$ th rotor shaft segment, orthogonal co-ordinates, generalized co-ordinates describing motion of the bearing housings in the vertical and horizontal direction,

eigenvector component for the generalized co-ordinate describing vertical vibrations of the bearing housings,

eigenvector component for the generalized co-ordinate describing horizontal vibrations of the bearing housings,

coefficient of the orthogonality properties (modal mass),

$\varepsilon_{i}$

$\varphi_{i}(t)$

$\Phi_{i}, \Psi_{i}$

$\mu_{i}$

$\omega, \omega_{m}$

$\Omega$

$\rho$

$\tau$

$\Theta_{i}(x)$

$\vartheta_{m}(t)$ axial distribution of the radial eccentricity of gravity centres of the $i$ th rotor shaft segment,

phase angles of the radial eccentricities of gravity centres of the rigid ring representing rotor and of the $i$ th rotor shaft segment, respectively,

$\phi_{i}(t), \psi_{i}(t)$

$\theta_{i}(x, t)$

$i, j, k, l, m, s$

radial eccentricity of the gravity centre of the rotor or disk, angular displacements in the vertical and horizontal plane of the rigid rings,

complex angular displacement of the rigid rings,

eigenvector components of the angular displacements in the vertical and horizontal plane for the rigid rings,

bending stiffness of the membranes connecting the rigid rings with the rotor shaft,

natural frequencies,

rotor shaft average rotational velocity,

material density,

torsional material viscosity,

angular displacement of the $i$ th rotor shaft segment,

eigenfunction of torsional displacements of the $i$ th rotor shaft segment,

Lagrange's co-ordinates for displacements in the vertical and horizontal plane,

Lagrange's coordinate for torsional displacements, indices.

\section{References}

Bently, D.E., Goldman, P. and Muszyńska, A., 1995. "Snapping" torsional response of an anisotropic radially loaded rotor. Papers of the ASME, 95-GT-396.

Bogacz, R., Szolc, T. and Irretier, H., 1992. An application of torsional wave analysis to turbogenerator rotor shaft 
response. ASME Tran., J. of Vibration and Acoustics, 114, 149-153.

Ecker, H., Springer, H. and Haselbacher, H., 1994. Dynamic response of a rotor bearing system due to bearing failure. In: Proc. of the 4th Int. IFToMM Conference on Rotor Dynamics, Chicago, ed., R.L. Eshleman, Vibration Institutè, USA, pp. $357-362$.

Gryboś, R., 1994. Rotor dynamics. Polish Scientific Publishers (PWN), Warsaw (in Polish).

Kim, P.Y., Flanagan, R.C. and Lowe, I.R.G., 1989. A new method for the critical speed calculation of the rotor-bearing systems - Part I and II. In: Proc. of the 12th Biennial ASME Conf. of Mechanical Vibration and Noise, Montreal, Rotating Machinery Dynamics, DE-Vol. 18-1, pp. 71-82.

Lee, C.W. and Jei, Y.-G., 1988. Modal analysis of continuous rotor-bearing systems. J. of Sound and Vibration, 126(2), $345-361$.

Muszyńska, A. and Goldman, P., 1995. Chaotic responses of unbalanced rotor/bearing/stator systems with looseness or rubs. Chaos, Solitons \& Fractals, 5(9), 1683-1704.

Neilson, R.D., 1992. Dynamics of electric submersible pumps. In: Proc. of the International Conference on Rotating Machine Dynamic, “Rotordynamics'92", Venice, Italy, ed., Michael, J. Goodwin, April 1992, Springer-Verlag, pp. 302-309.

Nelson, H.D., 1994. Modeling, analysis and computation in rotordynamics: a historical perspective. In: Proc. of the 4th Int. IFToMM Conference on Rotor Dynamics, Chicago, ed., R.L. Eshleman, Vibration Institute, USA, pp. 171-177.

Szolc, T., 1998. Medium frequency dynamic investigation of the railway wheelset-track system using a discrete-continuous model. Archive of Applied Mechanics - Ingenieur Archiv, 68(1), 30-45.

Tondl, A., 1965. Some Problems of Rotor Dynamics. Publishing House of the Czechoslovak Academy of Sciences, Prague.

\section{APPENDIX}

The coefficients of the orthogonality properties

- for system lateral motion in the vertical plane:

$$
\begin{aligned}
\gamma_{U m}^{2}= & \sum_{i=1}^{n+1}\left[m_{i} U_{i m}^{2}\left(L_{i}\right)+J_{i} \Phi_{i m}^{2}\right] \\
& +\rho \sum_{i=1}^{n} A_{i} \int_{L_{i}}^{L_{i+1}} U_{i m}^{2}(x) \mathrm{d} x \\
& +\rho \sum_{i=1}^{n} I_{i} \int_{L_{i}}^{L_{i+1}}\left(\frac{\mathrm{d} U_{i m}(x)}{\mathrm{d} x}\right)^{2} \mathrm{~d} x \\
& +\sum_{k} m_{\mathrm{B} k} Y_{k m}^{2},
\end{aligned}
$$

- for system lateral motion in the horizontal plane:

$$
\begin{aligned}
\gamma_{W m}^{2}= & \sum_{i=1}^{n+1}\left[m_{i} W_{i m}^{2}\left(L_{i}\right)+J_{i} \Psi_{i m}^{2}\right] \\
& +\rho \sum_{i=1}^{n} A_{i} \int_{L_{i}}^{L_{i+1}} W_{i m}^{2}(x) \mathrm{d} x \\
& +\rho \sum_{i=1}^{n} I_{i} \int_{L_{i}}^{L_{i+1}}\left(\frac{\mathrm{d} W_{i m}(x)}{\mathrm{d} x}\right)^{2} \mathrm{~d} x \\
& +\sum_{k} m_{\mathrm{B} k} Z_{k m}^{2},
\end{aligned}
$$

- for torsional motion of the rotor shaft:

$$
\gamma_{T m}^{2}=\sum_{i=1}^{n+1} I_{0 i} \Theta_{i m}^{2}\left(L_{i}\right)+\rho \sum_{i=1}^{n} J_{0 i} \int_{L_{i}}^{L_{i+1}} \Theta_{i m}^{2}(x) \mathrm{d} x
$$

where:

$$
\begin{aligned}
L_{i}= & \sum_{j=1}^{i-1} l_{j}, \quad i=1,2, \ldots, n+1, \\
& \Phi_{k m}=\frac{\mathrm{d} U_{k m}(x)}{\mathrm{d} x}, \quad \Psi_{k m}=\frac{\mathrm{d} W_{k m}(x)}{\mathrm{d} x} \\
& \text { for } x=L_{k}, \quad 1 \leq k \leq n, \quad m=1,2, \ldots
\end{aligned}
$$




\section{ait \\ ENERGY MATERIALS}

M A N E Y publishing

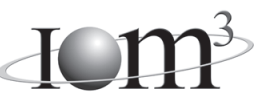

\section{Materials Science \& Engineering for Energy Systems}

Maney Publishing on behalf of the Institute of Materials, Minerals and Mining

The Institute of Materials, Minerals \& Mining

Economic and environmental factors are creating ever greater pressures for the efficient generation, transmission and use of energy. Materials developments are crucial to progress in all these areas: to innovation in design; to extending lifetime and maintenance intervals; and to successful operation in more demanding environments. Drawing together the broad community with interests in these areas, Energy Materials addresses materials needs in future energy generation, transmission, utilisation, conservation and storage. The journal covers thermal generation and gas turbines; renewable power (wind, wave, tidal, hydro, solar and geothermal); fuel cells (low and high temperature); materials issues relevant to biomass and biotechnology; nuclear power generation (fission and fusion); hydrogen generation and storage in the context of the 'hydrogen economy'; and the transmission and storage of the energy produced.

As well as publishing high-quality peer-reviewed research, Energy Materials promotes discussion of issues common to all sectors, through commissioned reviews and commentaries. The journal includes coverage of energy economics and policy, and broader social issues, since the political and legislative context influence research and investment decisions.

\section{CALL FOR PAPERS}

Contributions to the journal should be submitted online at http://ema.edmgr.com

To view the Notes for Contributors please visit: www.maney.co.uk/journals/notes/ema

Upon publication in 2006, this journal will be available via the Ingenta Connect journals service. To view free sample content online visit: www.ingentaconnect.com/content/maney

For further information please contact:

Maney Publishing UK

Tel: +44 (0)113 2497481 Fax: +44 (0)1132486983 Email: subscriptions@maney.co.uk

or

Maney Publishing North America

Tel (toll free): 8662975154 Fax: 6173546875 Email: maney@maneyusa.com

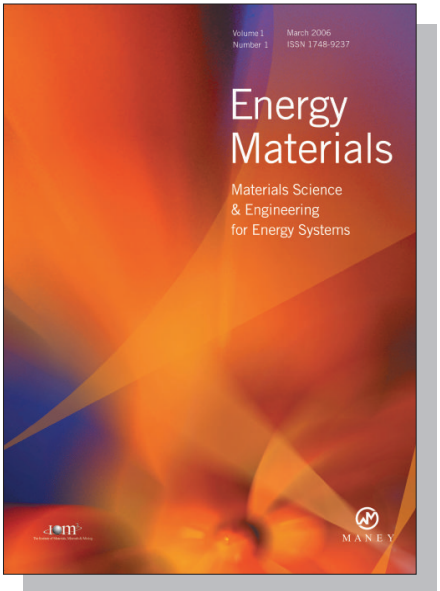

EDITORS

Dr Fujio Abe

NIMS, Japan

Dr John Hald, IPL-MPT, Technical University of Denmark, Denmark

Dr R Viswanathan, EPRI, USA

\section{SUBSCRIPTION INFORMATION}

Volume 1 (2006), 4 issues per year

Print ISSN: 1748-9237 Online ISSN: 1748-9245

Individual rate: $£ 76.00 / U S \$ 141.00$

Institutional rate: $£ 235.00 /$ US $\$ 435.00$

Online-only institutional rate: $£ 199.00 / U S \$ 367.00$

For special $\mathrm{IOM}^{3}$ member rates please email

subscriptions@maney.co.uk

\section{For further information or to subscribe online please visit www.maney.co.uk}



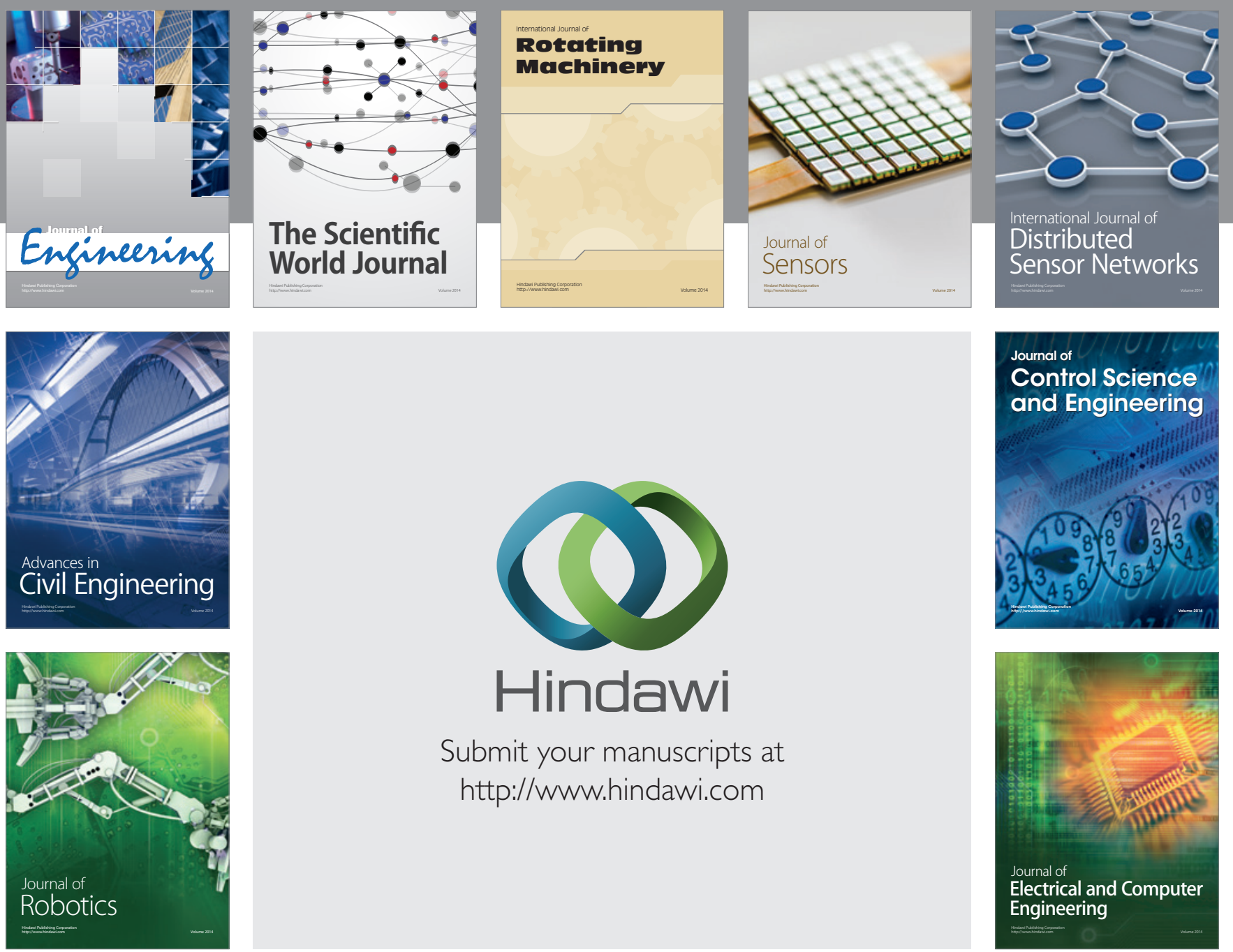

Submit your manuscripts at

http://www.hindawi.com
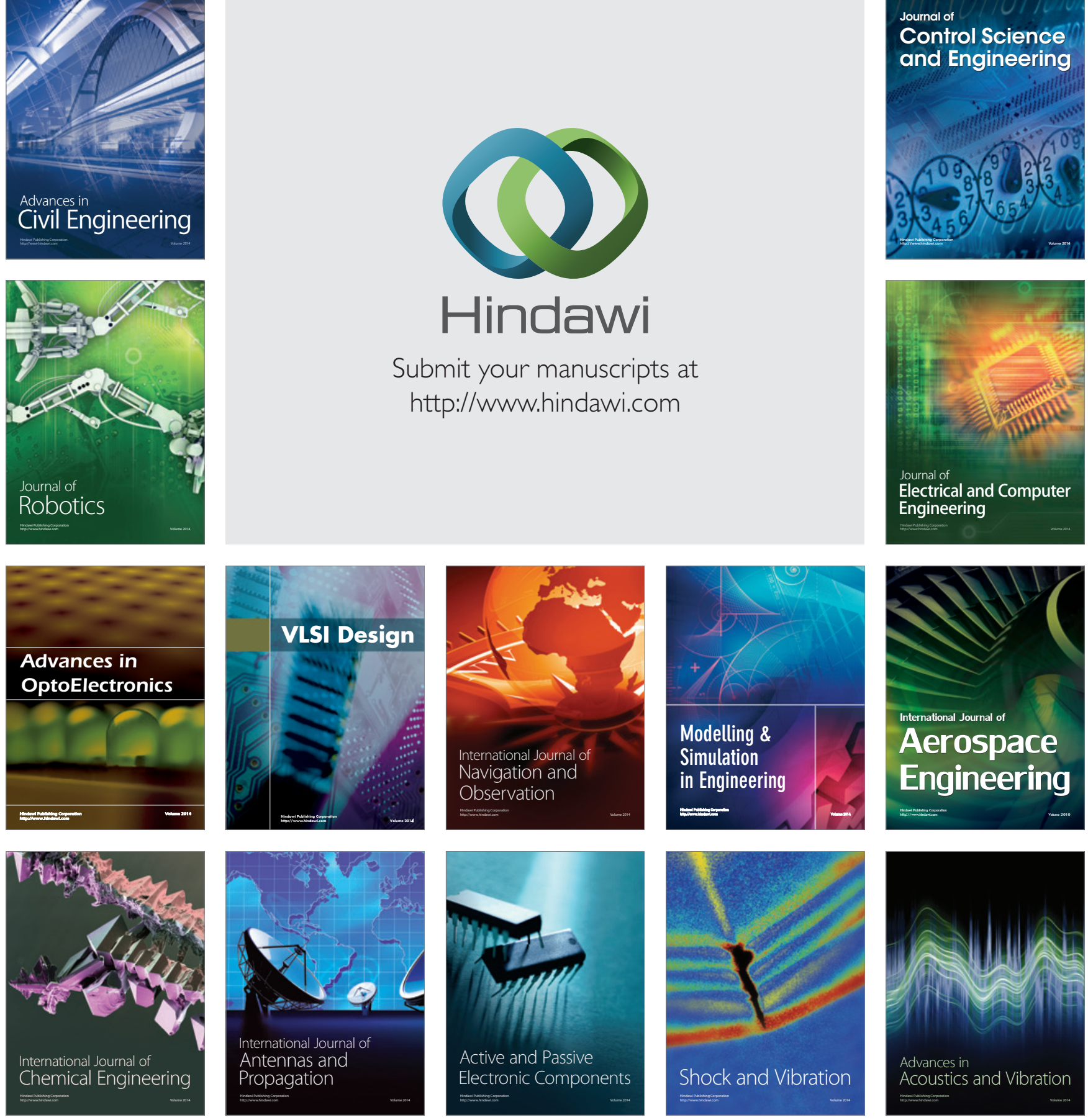